\title{
Impacts and Limits of Irrigation Water Management on Wheat Yield and Quality
}

\section{Jessica A. Torrion* and Robert N. Stougaard}

Montana State University- Northwestern Agricultural Research Center, 4570 MT 35, Kalispell, MT 59901

*jessica.torrion@montana.edu

Phone: 406-755-4303 Fax: 406-755-8951

Abbreviations: C, cultivar; EM, early milk; ET, evapotranspiration; ETc, crop ET; ETo, reference ET; FL, anthesis; FN, falling number; HI, harvest index; HRSW, hard red spring wheat; I, irrigation; $\mathrm{K}_{\mathrm{c}}$, crop coefficient, MM, medium milk; PHS, Pre-harvest sprouting; PM, physiological maturity; SPP, spikes plant ${ }^{-1}$; SPS, seeds spike ${ }^{-1}$; TKW, thousand kernel weight; TWT, grain test weight; Y, year.

This PDF file contains Supplemental Information. 

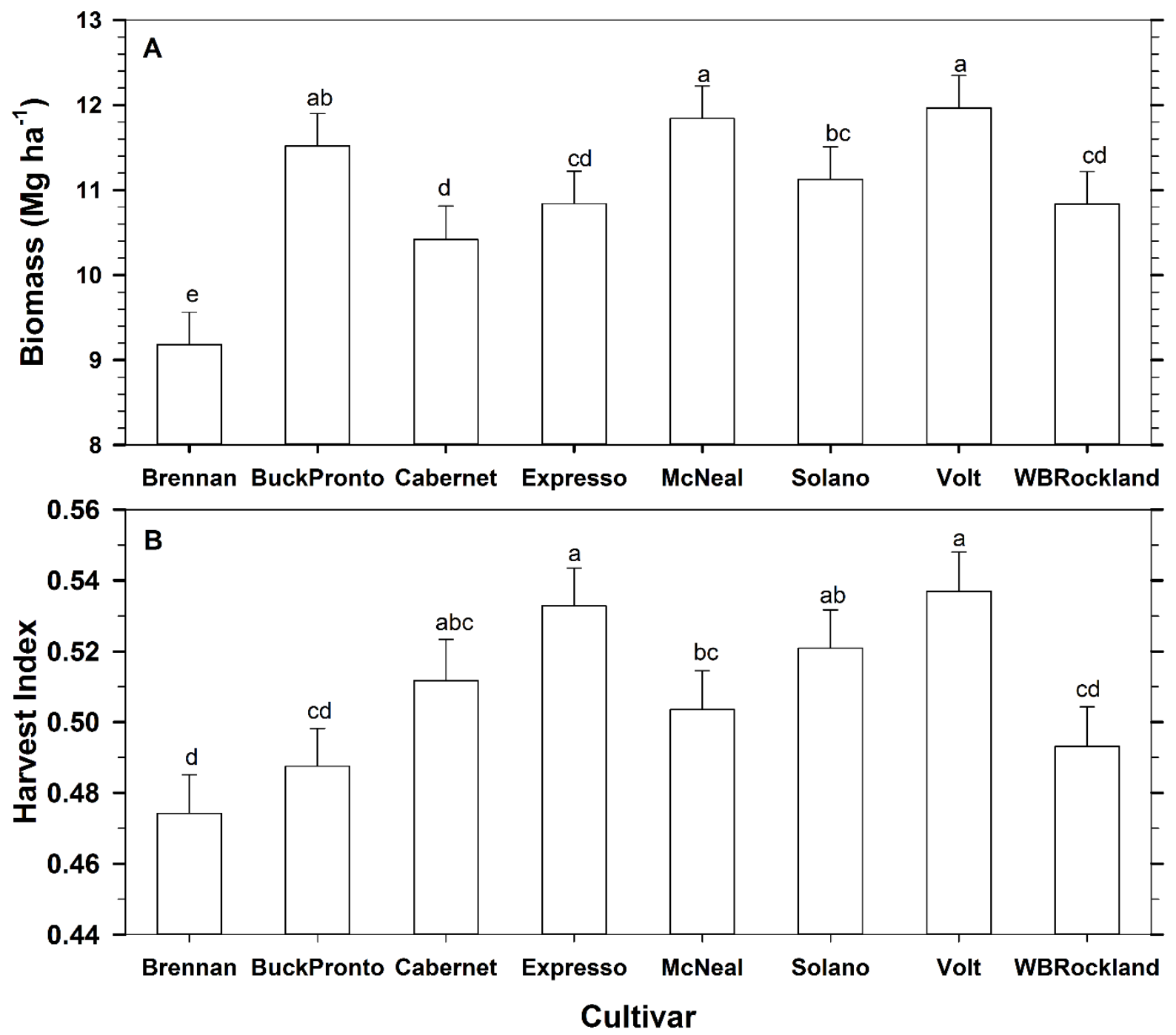

Supplemental Fig. S1. Cultivar means for aboveground biomass (Panel A) and harvest index (Panel B). Error bar shown is the standard error of the difference between cultivar treatment means. Same letter assignment indicates the nonsignificance between cultivar means at $\alpha=0.05$. 



Supplemental Fig. S2. Cultivar means for the respective years $(2014,2015)$ on yield (Panels A,

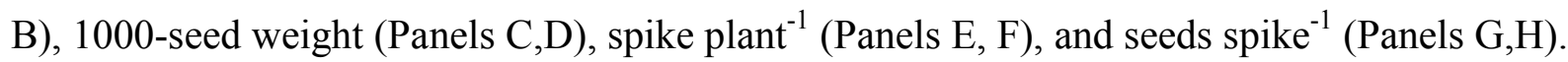
Error bar is the standard error of the difference between cultivar treatment means. Same letter assignment indicates the nonsignificance between cultivar means at $\alpha=0.05$. 

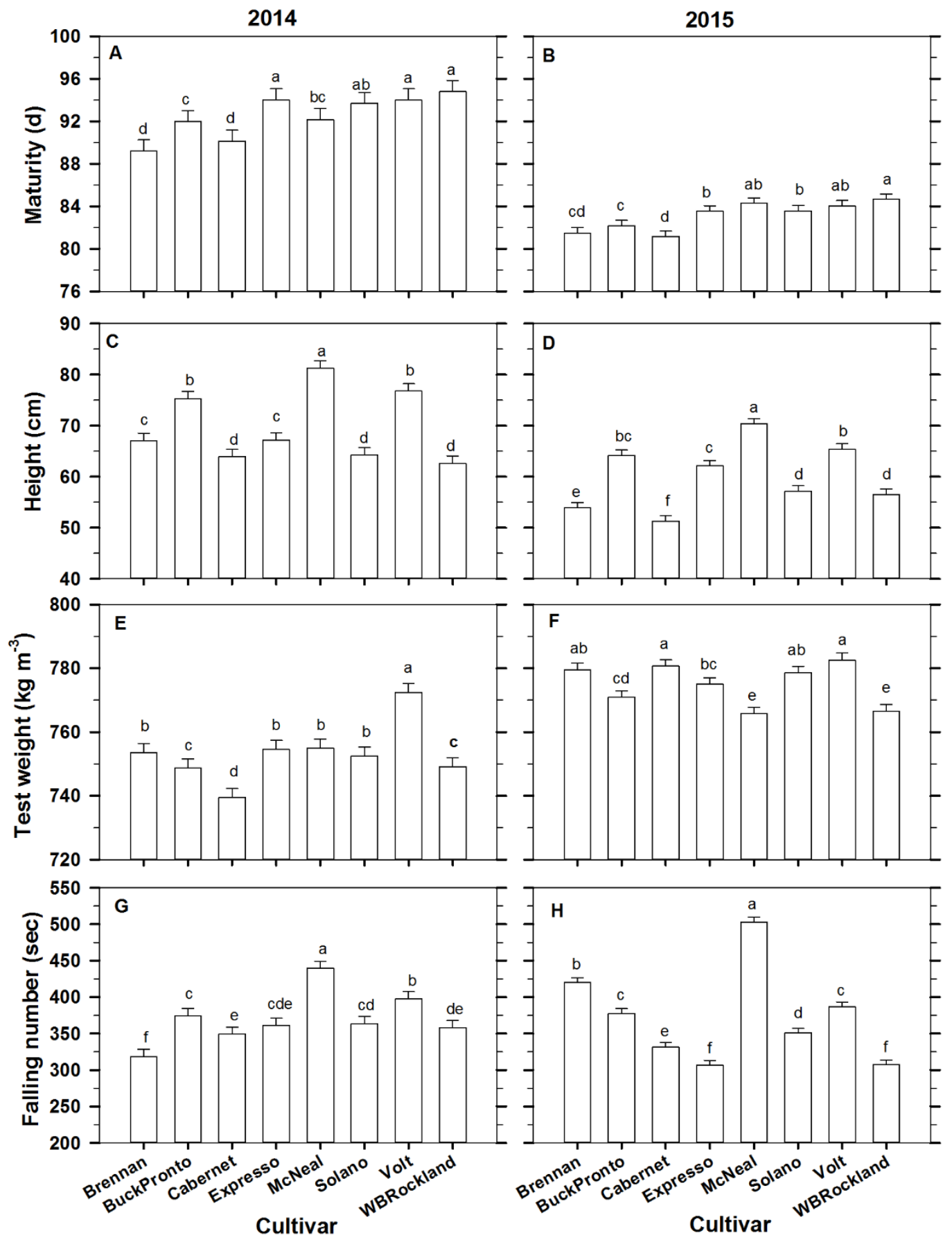

Supplemental Fig. S3. Cultivar means for the respective years $(2014,2015)$ on d to maturity (Panels A, B), height (Panels C,D), test weight (Panels E, F), and falling number (Panels G,H). Error bar is the standard error of the difference between cultivar treatment means. Same letter assignment indicates the nonsignificance between cultivar means at $\alpha=0.05$. 